\title{
Combining echocardiographic and anatomic variables to predict outcomes of mitral valve repair with the NeoChord procedure
}

\author{
Erica Manzan $^{1,2 \#}$, Danila Azzolina ${ }^{3 \#}$, Dario Gregori ${ }^{3}$, Eleonora Bizzotto ${ }^{4}$, Andrea Colli ${ }^{5}$, Gino Gerosa ${ }^{1}$ \\ ${ }^{1}$ Cardiac Surgery Unit, Department of Cardiac-Thoracic-Vascular Sciences and Public Health, University of Padua, Padua, Italy; ${ }^{2}$ Cardiac Surgery \\ Unit, A.O.R. San Carlo Hospital, Potenza, Italy; ${ }^{3}$ Unit of Biostatistics, Epidemiology and Public Health, Department of Cardiac-Thoracic-Vascular \\ Sciences and Public Health, University of Padua, Padua, Italy; ${ }^{4}$ Cardiology Unit, Policlinico di Abano Terme, Abano Terme, Italy; ${ }^{5}$ Cardiac Surgery \\ Unit, Department of Surgical, Medical and Molecular Pathology and Critical Care, University of Pisa, Pisa, Italy \\ "These authors contributed equally to this work. \\ Correspondence to: Prof. Gino Gerosa. Department of Cardiac-Thoracic-Vascular Sciences and Public Health, University of Padua, Via Giustiniani 2, \\ 35128 Padova, Italy. Email: gino.gerosa@unipd.it.
}

Background: Trans-apical, echo-guided NeoChord mitral valve (MV) repair is an innovative procedure to treat degenerative mitral regurgitation (MR) without concomitant annuloplasty. Recently, leaflet-to-annulus index (LAI) has been identified as a positive prognostic predictor of outcomes at 1-year follow up. The aim of this study is to develop a pre-operative predictor tool to assess probability of success with NeoChord procedure utilizing multi-factor echocardiographic and anatomic variables.

Methods: We included ninety-one consecutive patients with prolapse/flail of the posterior mitral leaflet, who subsequently underwent NeoChord MV repair between November 2013 and October 2016. All patients completed post-operative echocardiographic follow-up assessments for up to 2 years. A random forest regression algorithm identified and ranked the most relevant predictors of moderate-severe MR. A multi-variable Cox regression model was performed at follow-up intervals, to assess variables associated with residual MR that was classified as mild or less. Bootstrapping re-samples were used to validate an estimated survival model. Predictive accuracy was assessed using a discrimination index that corrected for overoptimism.

Results: We developed a nomogram which used the results of a multi-variable model to predict the probability of mild or less residual MR at follow-up periods (discharge, 1, 3, 6 months, 1 and 2 years). Identified predictors included LAI, systolic pulmonary artery pressure, indexed left ventricle end-systolic volume (iLVESV), prolapse/flail width (FW), systolic antero-posterior (AP) annulus diameter, systolic laterolateral (LL) annulus diameter and presence of calcification.

Conclusions: A NeoChord MV repair prediction tool would be helpful in clinical decision-making and in the identification of patients who may benefit from a ringless mitral valve repair using the NeoChord procedure.

Keywords: Mitral valve regurgitation (MV regurgitation); off-pump; transapical; mitral valve repair (MV repair); neochordae

Submitted May 20, 2020. Accepted for publication Nov 20, 2020.

doi: $10.21037 / \mathrm{acs}-2020-\mathrm{mv}-96$

View this article at: http://dx.doi.org/10.21037/acs-2020-mv-96 


\section{Introduction}

Trans-apical, echo-guided mitral valve (MV) repair using the NeoChord procedure has been shown to be safe and effective in correcting degenerative mitral regurgitation (MR) without concomitant annuloplasty (1-4). The NeoChord procedure has been standardized and is of reproducible quality.

Recently, leaflet-to-annulus index (LAI) [ratio between the sum of anterior leaflet length (AML) and posterior leaflet length (PML) over antero-posterior (AP) length, $(\mathrm{AML}+\mathrm{PML}) / \mathrm{AP}]$ has been reported as a positive prognostic predictor of follow-up outcomes at 1 year (5). A more recent analysis of the failure mechanisms of the NeoChord procedure have inferred that, notwithstanding technical causes, anatomical aspects of the MV and left ventricle $(\mathrm{LV})$ should be pre-operatively considered when selecting candidates for this innovative procedure (6). In our practice, during the pre-operative screening process, we typically review multiple echocardiographic parameters, as well as the LAI. However, consideration of all individual variables is associated with a mental algorithm of sorts and subjective decision-making based on surgical experience rather than objective assessment of the combined parameters. The intent of a nomogram is to create an objective method with which to predict procedural success at follow-up, based on interpretation of the continuous and categorical echocardiographic and anatomical variables associated with a continuous series of treated patients.

The aim of this study is to develop a pre-operative assessment tool that can be used to predict success of the NeoChord procedure at follow-up, with the utilization of multi-factorial echocardiographic and anatomic variables.

\section{Methods}

All consecutive patients with an isolated, central segment (P2) prolapse/flail and patients with multi-segment posterior leaflet disease, who underwent NeoChord procedure between November 2013 and October 2016, were included in this study. All patients completed postprocedure echocardiographic assessment for up to 2 years. The study protocol conforms to the ethical guidelines of the 1975 Declaration of Helsinki, as reflected by the institution's human research committee approval.

\section{Neochord procedure}

The Neochord procedure was performed in standard cardiac surgery operating theatres, with the patient placed under general anaesthesia and mechanically ventilated through a double-lumen endotracheal tube. An intraoperative cell-saver system was used. Stand-by cardiopulmonary bypass was ensured and a safety-net preparation of the femoral vessels was obtained.

The NeoChord procedure was performed through a left lateral mini-thoracotomy in the fifth intercostal space. Real-time 2D and 3D transesophageal echocardiography (TEE) guided the procedure according to standardized protocol $(7,8)$.

The most appropriate ventricular access site was manually identified on the postero-lateral wall of the $\mathrm{LV}$ with the "finger test", under real time 2D-TEE X-plane imaging (obtained from the mid-esophageal long-axis and mitral commissure views). Two concentric purse string sutures with pledgets were placed. The NeoChord DS1000 was introduced in the $\mathrm{LV}$ and guided across the MV using 2D real-time TEE $X$-plane views. Once the $M V$ was crossed, the echocardiography was shifted to $3 \mathrm{D}$ surgical views, guiding the surgeon to the deployment of the neochord on the appropriate diseased leaflet and segment of the MV. When a correct position was achieved, the jaws of the device were opened and the leaflet was grasped. A good grasp of sufficient leaflet tissue was confirmed when all four fibre optic monitor lights turned from red to white. Once the chordae was implanted, the device was retrieved from the $L V$ and a hitch knot was performed with the Goretex suture in order to secure the chordae to the leaflet. The sequence of implantation was then repeated as many times as necessary to reach good MV coaptation. The purse strings were tightened and the neochordae were passed through a large Teflon pledget, placed over the ventriculotomy. Finally, each neochord was independently tensioned with tourniquets to obtain good coaptation of the leaflets and significant reduction of the residual MR.

\section{Statistical analysis}

Descriptive statistics were used to characterize patients involved in the study. Categorical data were reported as relative and absolute frequencies; continuous data as mean and standard deviation.

Random Forest regression was performed to identify the most relevant predictors of moderate and severe $M R$ at follow-up (9). A variable importance metric plot (VIMP) was utilized, as reported in literature, to assess the ranking of these predictors (10). Once the relevant predictors had 
been identified, a prognostic model for procedural success was defined using a multivariable Cox regression analysis, in order to identify moderate and severe MR hazards at follow-up periods. The estimated survival model has been internally validated using bootstrap re-sampling procedures (500 samples). The predictive accuracy of the model has been assessed using the Harrell-C discrimination index and corrected for over-optimism (11). It is generally accepted that Harrell-C values of $0.7-0.8$ represent reasonable discrimination, and those values exceeding 0.8 represent a good level of discrimination (12).

In regards to the continuous predictors, the hazard ratios (HRs) estimates have been reported according to interquartile range (IQR), indicating the estimated variation in the $>$ mild MR hazard as a result of a predictor increase, ranging from the first to the third quartile.

We developed the nomogram for use as a graphical tool, utilizing the results of the multivariable model for predicting the probabilities of residual $\leq$ mild MR at different follow-up times (discharge, 1, 3, 6 months, 1 and 2 years). Predictors used in the model were: LAI, flail width $(\mathrm{FW})$, presence of calcification, entry site, systolic AP annulus; systolic latero-lateral (LL) annulus, indexed left ventricle end-systolic volume (iLVESV), systolic pulmonary artery pressure (sPAP). LAI and prolapse/FW were defined and measured as previously described (Figure 1) (5) using dedicated software [4D MV-Assessment, TomTec Imaging Systems (GmbH, Munich, Germany)]. Coaptation length index (CL) was calculated according to the Yoshida's formula (13). At the follow-up periods, MR severity was graded as absent/trace (0), mild (1+), moderate $(2+)$, or severe $(3+)$ based on a combination of semi-quantitative and qualitative parameters (14). Entry site evaluation was described as normal or anterior, as previously published (15). We performed statistical computations using $\mathrm{R}$, version 3.5.2 (16) with RMS and survival packages (17).

\section{Results}

\section{Demographic and echocardiographic data}

The study cohort ( $\mathrm{N}=91$ patients) was composed primarily of male subjects $(82 / 91,90 \%)$, with a mean age of $65 \pm 13$ years and with a mean EuroSCORE II of $2.06 \% \pm 3.18 \%$. Sinus rhythm was observed in $92 \%$ (84/91) of subjects. Echocardiography demonstrated slightly dilated left ventricles in the cohort, with mean iLVESVs of $32.9 \pm$ $10.6 \mathrm{~mL} / \mathrm{m}^{2}$ and mean indexed left ventricle end-diastolic volumes (iLVEDV) of $81 \pm 17.7 \mathrm{~mL} / \mathrm{m}^{2}$, with mean systolic $\mathrm{AP}$ diameter, mean LL diameter and mean $\mathrm{FW}$ reported as $38.3 \pm 5.8,38.3 \pm 5.3$, and $7.7 \pm 5.2 \mathrm{~mm}$, respectively. Residual MR at follow-up is presented in Figure 2.

At 2-year follow-up, no MR was observed in 32 patients (35.2\%), mild MR in 36 (39.5\%), moderate MR in 20 $(22 \%)$ and severe $M R$ in $3(3.3 \%)$ patients. In one case of Type A MR, recurrence of MR $>2+$ was due to AML pseudo-prolapse for an excessive tensioning of implanted neochordae, not followed by the expected LV remodeling. The patient was in good clinical condition (NYHA Class I), and therefore, it was decided to delay re-intervention. Another patient was an 80 -year-old female with complex MV anatomy (Type D, with a cleft between P1 and $\mathrm{P} 2$ prolapsing segments, AML tenting and annulus calcifications) and severe pulmonary hypertension. She was deemed inoperable with conventional surgical methods, due to severe comorbidities (EuroScore II 24.87\%, STS-PROM for $M V$ repair $8.52 \%$ ). Therefore, she was medically treated and stabilized in NYHA Class II. The last patient (Type B) with recurrence of severe MR, presented a central jet due to LV and annulus dilation, with NYHA Class II under optimal medical therapy.

All successfully treated patients showed a significant improvement in NYHA functional class. At 2-year followup, 83 patients $(91.2 \%)$ were in NYHA Class I, and eight $(8.8 \%)$ in NYHA II.

\section{Predictive model}

The predictive model was defined using the VIMP metric and computed via a Random Forest algorithm. The top five predictors were identified via the VIMP ranking curve (Figure 3). Two additional covariates, systolic AP annulus diameter and iLVESV, were selected as predictive variables based on their clinical importance in both pre-operative and post-operative assessments. The predictive variables identified for the model include: LAI, prolapse/FW, systolic $\mathrm{AP}$ and LL mitral annular dimensions, presence of valve calcification, iLVESV and sPAP.

Once the predictors were identified, Cox regression analysis was computed and confirmed that the hazard of moderate-severe MR is increased when associated with increases in sPAP $(\mathrm{mmHg})$, prolapse/FW, systolic LL annulus dimension and with the presence of calcification (Table 1). Additionally, a Harrell-C discrimination index was computed to correct for over-optimism. Based on 500 


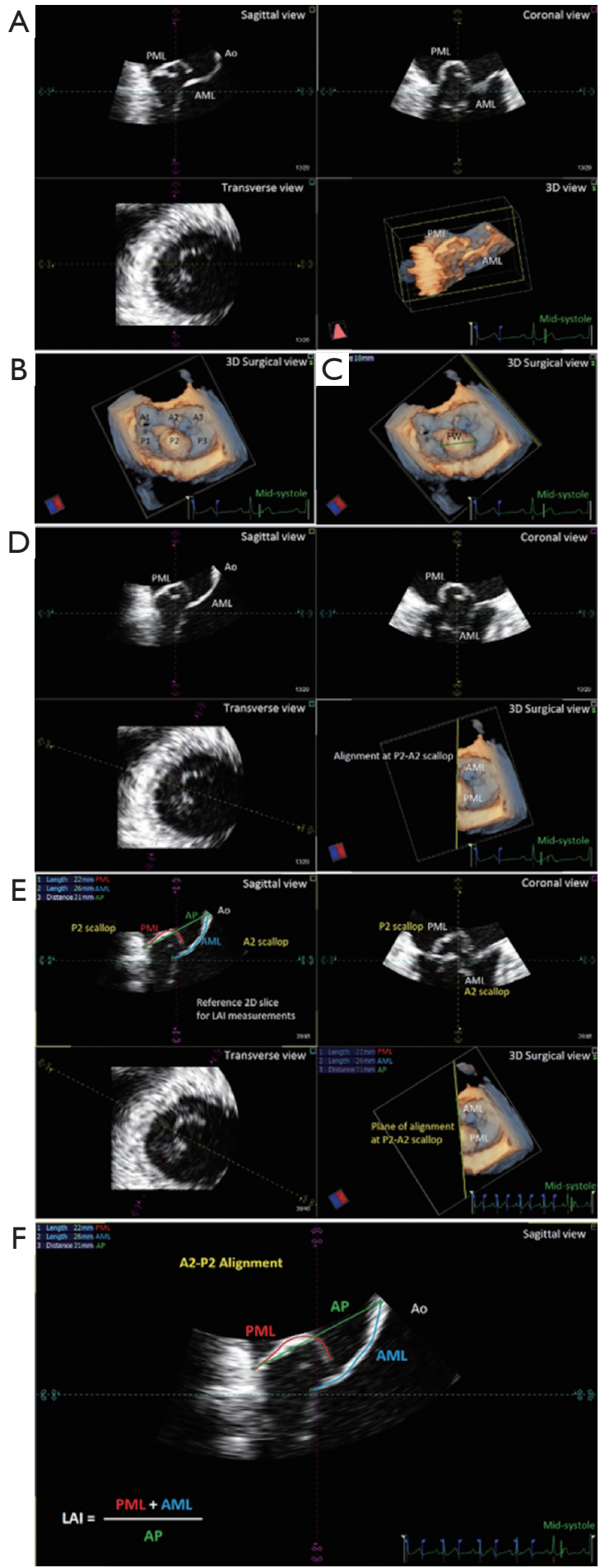

Figure 1 Leaflet-to-annulus measurement. (A) In the 4D Cardio-View interface, the sagittal, coronal and transverse views are displayed simultaneously in MPR, with the rendered 3D image in the lower right quadrant; (B) the 3D surgical view of the MV is obtained; (C) FW measurement is performed using 3D surgical view; (D) MPR alignment of the MV is obtained; (E) AML, PML and AP measurements were performed on 2D sagittal view in mid-systole, at the level of P2/A2 segments; (F) example of LAI calculation. MPR, multiplane reconstruction; MV, mitral valve; AML, anterior mitral leaflet; PML, posterior mitral leaflet; FW, flail width; AP, antero-posterior annulus diameter; LAI, leaflet-to-annulus index; Ao, aorta. 


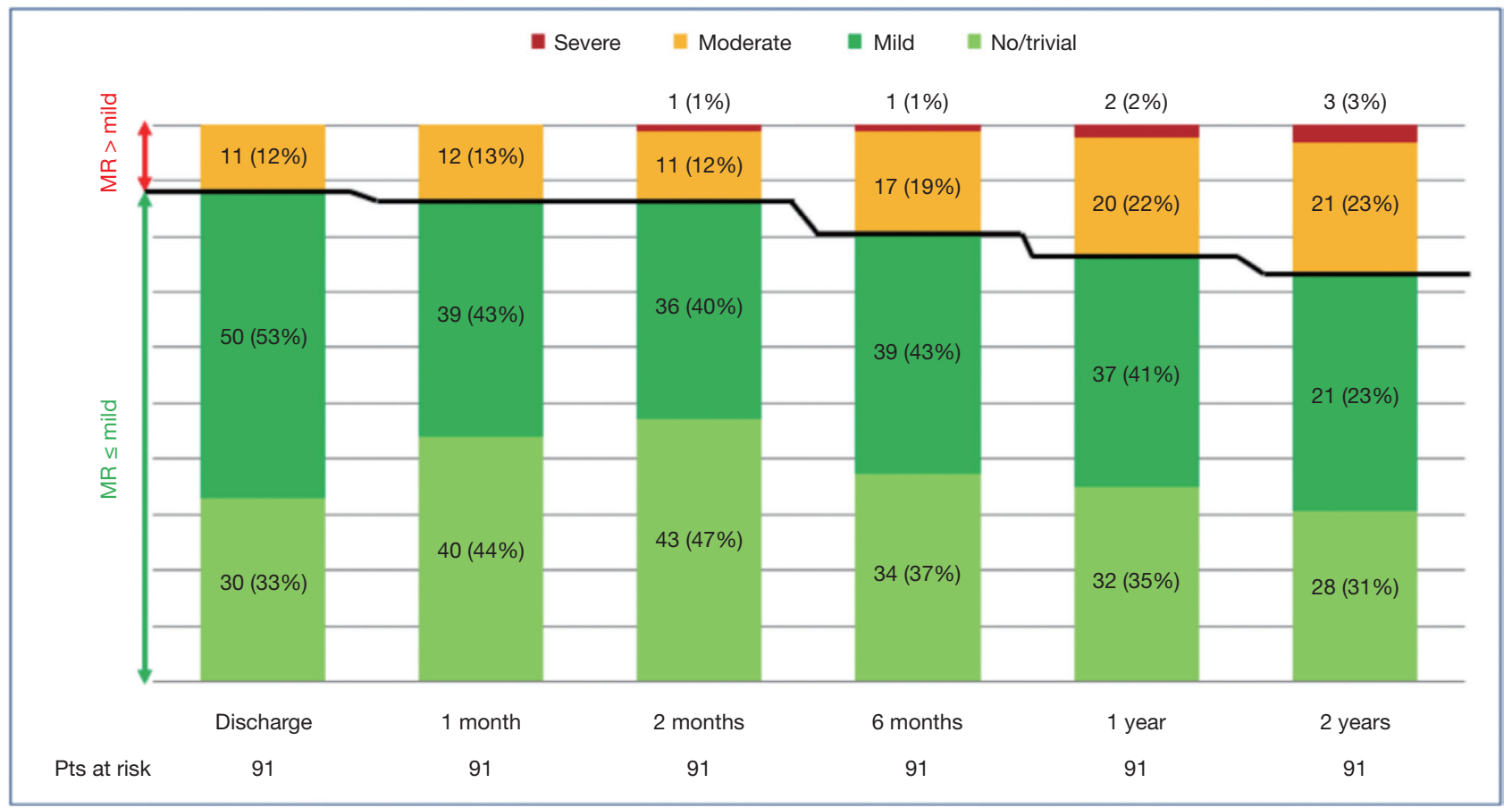

Figure 2 Echocardiographic outcome at follow up. According to the grade of residual MR, the population was divided in two groups (MR $>1+v s . M R \leq 1+)$. MR, mitral regurgitation; pts, patients.
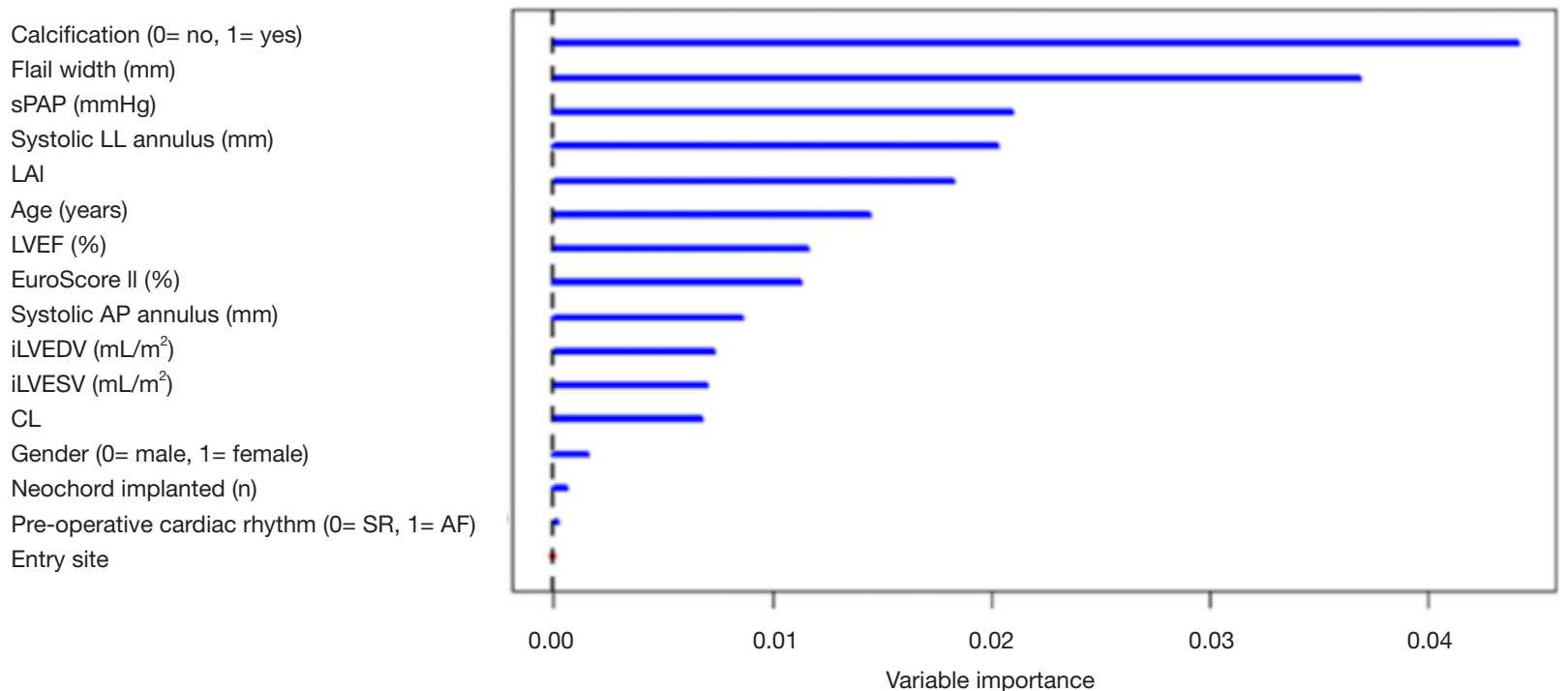

Figure 3 Random forest VIMP. VIMP, variable importance metric plot; sPAP, systolic pulmonary artery pressure; LL, latero-lateral; LAI, leaflet-to-annulus index; LVEF, left ventricle ejection fraction; AP, antero-posterior; iLVEDV, indexed left ventricle end-diastolic volume; iLVESV, indexed left ventricle end-systolic volume; CL, coaptation length index; SR, sinus rhythm; AF, atrial fibrillation. 


\begin{tabular}{|lllll}
\hline \multicolumn{2}{l}{ Table 1 Multivariable Cox model estimates moderate-severe MR HR } & & & Upper bound \\
\hline Variables [IQR] & HR & Lower bound & 1.18 & P value \\
\hline LAI [1.27-1.4] & 0.75 & 0.48 & 2.07 & $<.21$ \\
\hline SPAP (mmHg) [25-44] & 1.56 & 1.17 & 1.00 & 0.001 \\
\hline iLVESV (mL/m²) [23-39] & 0.71 & 0.49 & 1.98 & 0.03 \\
\hline FW (mm 3D) [14-21] & 1.43 & 1.03 & 1.25 & 0.3 \\
\hline Systolic AP annulus (mm) [35-42] & 0.78 & 0.48 & 4.04 & $<0.001$ \\
\hline Systolic LL annulus (mm) [34-41] & 2.46 & 1.50 & 9.17 & $<0.001$ \\
\hline Calcification (yes) & 4.90 & 2.62 & \\
\hline
\end{tabular}

For quantitative variables, HRs have been presented with reference to the IQR. For example, a sPAP increase from 25 (first quartile) to $44 \mathrm{mmHg}$ (third quartile) involves a $56 \%$ increase in the MR > mild hazard (HR: 1.56, 95\% Cl: 1.17-2.07). The 95\% Cls have been also reported. MR, mitral regurgitation; HR, hazard ratio; IQR, inter-quartile range; LAI, leaflet-to-annulus index; sPAP, systolic pulmonary artery pressure; iLVESV, indexed left ventricle end-systolic volume; FW, flail width; AP, antero-posterior; LL, latero-lateral; CI, confidence interval.

bootstrap re-samples, an index value 0.8 was achieved, indicating the positive nature of the model's predictive accuracy.

\section{Nomogram}

The accuracy of the predictive nomogram is presented in Figure 4. A point system was used to assign each echocardiographic predictor variable with point ranges from 0 to 100 . Total-point ranges from 0 to 220 were computed using the sum of points assigned to each echocardiographic variable. The nomogram plot displays the predicted probabilities of residual $\mathrm{MR} \leq$ mild at each follow-up (discharge, 1, 3, 6 months, 1 and 2 years) as a linear projection of the total calculated score.

\section{Discussion}

Surgical referral for MV repair is suggested for (I) patients presenting with symptomatic severe MR, (II) asymptomatic patients presenting with cardiac factors predicting a worse post-operative outcome such as left ventricle ejection fraction (LVEF) $\leq 60 \%$, left ventricle end-systolic diameter (LVESD) $\geq 45 \mathrm{~mm}$, new onset atrial fibrillation, sPAP $\geq 50 \mathrm{mmHg}$, with a general trend towards earlier referral in order to prevent unfavorable cardiac remodeling. NeoChord MV repair is a technique that provides an isolated leaflet repair treatment for patients suffering from degenerative MR. MV repair with the NeoChord procedure has demonstrated good clinical outcomes up to 1-year follow-up in a selected group of patients presenting with prolapse or flail of the posterior leaflet and without evidence of leaflet-to-annulus mismatch (4). Strict attention to patient selection is crucial for a successful repair with the NeoChord procedure.

Our initial experience with the NeoChord procedure dates back to early feasibility studies. As with many new medical procedures, with time and use, aspects of the procedure become better characterized and specific procedural techniques become standardized. With the NeoChord procedure, technical refinements included ventricular access site location, ventricular navigation protocol, single pledget, epicardial chordal fixation, chordal tensioning with the use of tourniquets, dynamic chordal tensioning techniques $(7,18)$ and the routine use of objective patient selection criteria such as anatomical classification and $\operatorname{LAI}(19,20)$.

To establish objective patient selection criteria, we first introduced a patient classification scheme based on anatomical assessment of the MV and extent of the MV disease. Type A patients presented with isolated, central segment prolapse/flail of the posterior leaflet; Type B patients presented with multi-segment, prolapse/flail of the posterior leaflet; Type $C$ patients presented with anterior leaflet or bi-leaflet prolapse/flail; Type D patients presented with para-commissural disease and/or leaflet/ annular calcification. Patients classified as Type A and B have been demonstrated to be the best suited candidates for the NeoChord procedure given the early stage of their MV disease. Patients classified as Type $\mathrm{C}$ or D are considered 


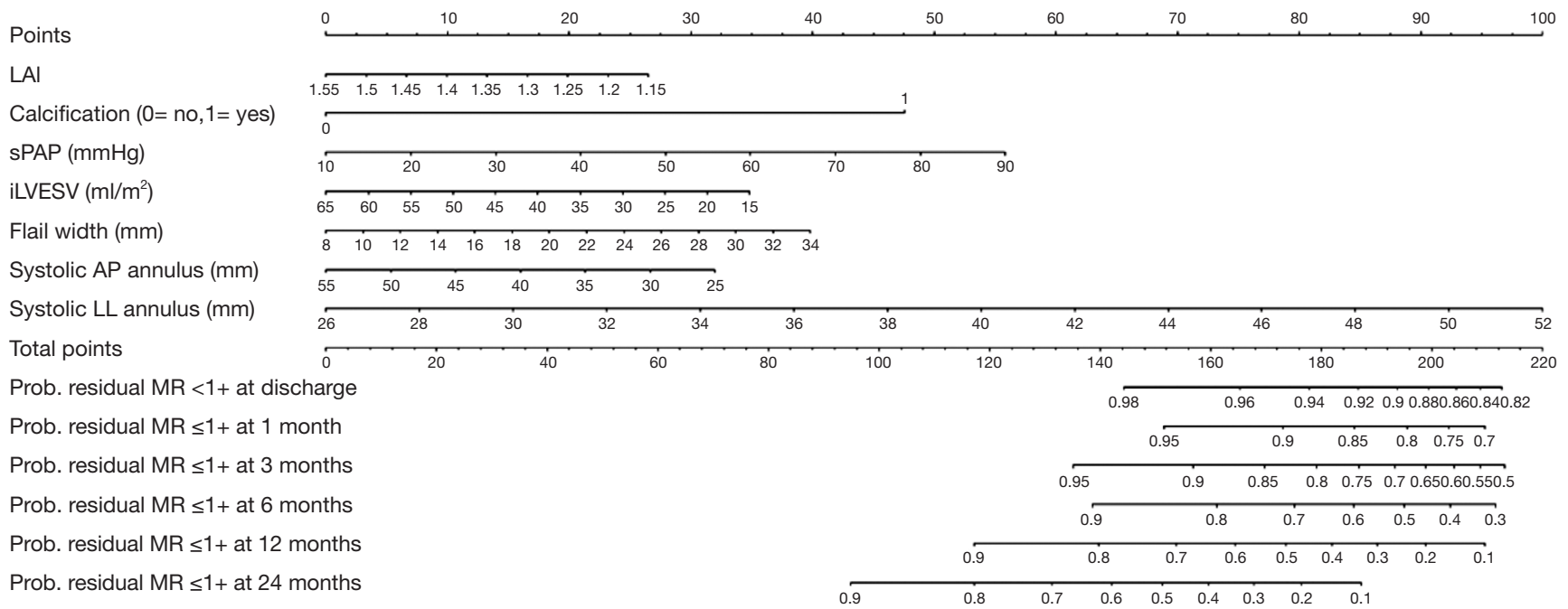

Figure 4 Nomogram for predicting moderate-severe MR free survival. To obtain the predicted probability of presenting MR less or equal to mild at follow-up, locate patient echocardiographic values at each axis. Draw a vertical line upward to the "points" axis to determine the points of each variable. Sum the points for all variables and locate the sum on the "total points" axis. Draw a vertical line down to the "probability of MR $\leq 1+$ at follow up" axis to find the patient's probability of presenting MR less or equal to Mild at each follow-up (discharge, 1, 3, 6, 12, 24 months after the procedure). Example: the ideal candidate for NeoChord repair would have a P2 prolapse with a LAI of 1.31 (points 17), a FW of $16 \mathrm{~mm}$ (points 13), no pulmonary hypertension (sPAP $20 \mathrm{mmHg}=$ points 7), no annulus or left ventricle dilation (systolic LL annulus $35 \mathrm{~mm}$ = points 35; systolic AP annulus $35 \mathrm{~mm}=$ points 21 ; iLVESV $30 \mathrm{~mL} / \mathrm{m}^{2}=$ points 24) and no calcification. With a total score of 117 , the patient will have more than $90 \%$ probability of $M R \leq 1+1$-year after NeoChord repair and more than $85 \%$ of $M R \leq 1+2$-year after Neochord procedure. Nomogram online version is available at the following link: https://r-ubesp.dctv.unipd.it/shiny/neochord/. MR, mitral regurgitation; LAI, leaflet-to-annulus index; FW, flail width; sPAP, systolic pulmonary artery pressure; LL, latero-lateral; AP, anteroposterior; iLVESV, indexed left ventricle end-systolic volume; Prob, probability.

more challenging patients for the NeoChord procedure, given the more complex valvular pathology and a more progressed disease stage (19), which also reflects the general trend of patients treated with traditional MV repair surgery.

Next, the introduction of the LAI index allowed us to further stratify our identification of potential candidates for the NeoChord procedure by employing a principle of leaflet-to-annulus mismatch (5). LAI effectively estimates the amount of leaflet tissue redundancy that is available to provide for a surface area of coaptation, post-operatively. All leaflet and annular lengths are evaluated in mid-systole, using the 2D-TEE long axis views with simultaneous three-dimension multi-planar reconstruction (3D-MPR) to confirm the mid-portion $\mathrm{A} 2 / \mathrm{P} 2$ segment alignment. It is important to underline that the use of $2 \mathrm{D}$ images for this assessment represents a potential advantage for the clinical application of this nomogram tool, due to its routine use and worldwide availability (Figure 1).

Throughout our continuous learning process with this innovative, ringless MV repair procedure, we have learned that there are other clinical, anatomical and technical elements that should be taken into account during a preclinical screening assessment of NeoChord procedure candidates. In particular, we have observed that width of the prolapse/flail segment, presence of calcification, AP and LL mitral annular dimensions, LV dimensions, as well as the presence of pulmonary hypertension have played a significant role in predicting post-operative outcomes. Some of these parameters describe MV morphology such as LAI, prolapse/FW, annulus dimensions and presence of calcifications, while other parameters define the stage of the MV disease, including LV dilation and pulmonary hypertension.

The objective of the NeoChord nomogram is to collectively utilize each of the aforementioned parameters to assist in improving the patient selection process for potential NeoChord procedure candidates for both experienced centers, as well as for emerging centers commencing this innovative MV repair program. The NeoChord nomogram is a plot that displays the predicted 
probability of achieving $\leq$ mild MR at follow-up and is intended for support in the patient selection decisionmaking process. The nomogram is a prognostic tool that predicts the probability of a successful MV repair with the NeoChord procedure at early and late follow-up periods. Therefore, the nomogram could assist in the patient selection process of determining whether apatient is an ideal candidate for the NeoChord procedure or would be better suited for a conventional MV repair. Obviously, predictive models only supplement clinical decision-making and do not replace good clinical judgment and/or patient preference. They may be useful in providing additional consideration points in patient screening processes. In contemporary practice, prediction tools that are based on statistical models, incorporating multiple prognostic variables, offer a far more accurate means of estimating outcomes. In particular, the use of nomograms has gained favor among clinicians due to greater accuracy, ease of use and ability to generate outcomes tailored to the individual patient.

In order to make the NeoChord nomogram easily available and more user-friendly, we have created a free online version of the prediction tool as an application-based calculator that can be accessed at https://r-ubesp.dctv.unipd. $\mathrm{it} /$ shiny/neochord/. On the home page of the application, a detailed description of how to calculate echocardiographic parameters is also available. In the application, variables can be manually entered or automatically generated using the specific formula. As previously described, the application calculates the predicted probability of residual $\leq$ MR mild at each follow-up time point, and is computed with a $95 \%$ confidence interval. An advantage of the application is that it will support the collection of anonymized clinical and echocardiographic data from different users around the world and consequently improve the validity of the current tool through a continuous update of the predictive algorithm based on reported collaborative results from users on a global scale.

Some limitations warrant consideration when interpreting the findings of this study, such as the observational nature of this study, the single center experience and the limited number of patients. The need for additional studies is warranted.

In conclusion, the NeoChord nomogram is a useful predictive tool to guide the pre-operative assessment and appropriate selection of patients who may be successfully treated with MV repair using the NeoChord procedure.

\section{Acknowledgments}

Funding: 4D MV-Assessment, TomTec Imaging Systems (GmbH, Munich, Germany) was acquired based on the grant GR-2010-2320784 from the Italian Ministry of Health.

\section{Footnote}

Conflicts of Interest: AC, EM, EB and GG received travel grants from NeoChord, Inc. (modest relationship). The other authors have no conflicts of interest to declare.

Open Access Statement: This is an Open Access article distributed in accordance with the Creative Commons Attribution-NonCommercial-NoDerivs 4.0 International License (CC BY-NC-ND 4.0), which permits the noncommercial replication and distribution of the article with the strict proviso that no changes or edits are made and the original work is properly cited (including links to both the formal publication through the relevant DOI and the license). See: https://creativecommons.org/licenses/by-nc-nd/4.0/.

\section{References}

1. Colli A, Manzan E, Rucinskas K, et al. Acute safety and efficacy of the NeoChord proceduret. Interact Cardiovasc Thorac Surg 2015;20:575-80; discussion 580-1.

2. Colli A, Manzan E, Zucchetta F, et al. Transapical offpump mitral valve repair with Neochord implantation: Early clinical results. Int J Cardiol 2016;204:23-8.

3. Colli A, Manzan E, Aidietis A, et al. An early European experience with transapical off-pump mitral valve repair with NeoChord implantation. Eur J Cardiothorac Surg 2018 Sep 1;54:460-6.

4. Colli A, Manzan E, Besola L, et al. One-year outcomes after transapical echocardiography-guided mitral valve repair. Circulation 2018;138:843-5.

5. Colli A, Besola L, Montagner M, et al. Prognostic impact of leaflet-to-annulus index in patients treated with transapical off-pump echo-guided mitral valve repair with NeoChord implantation. Int J Cardiol 2018;257:235-7.

6. Colli A, Besola L, Bizzotto E, et al. Mechanisms of recurrent regurgitation after transapical off-pump mitral valve repair with neochord implantation $\dagger$. Eur J Cardiothorac Surg 2019;56:479-87.

7. Colli A, Zucchetta F, Torregrossa G, et al. Transapical offpump mitral valve repair with Neochord Implantation 
(TOP-MINI): step-by-step guide. Ann Cardiothorac Surg 2015;4:295-7.

8. Colli A, Manzan E, Fabio FZ, et al. TEE-guided transapical beating-heart neochord implantation in mitral regurgitation. JACC Cardiovasc Imaging 2014;7:322-3.

9. Ishwaran H, Kogalur UB, Blackstone EH, et al. Random survival forests. Ann Appl Stat 2008;2:841-60.

10. Ishwaran H. Variable importance in binary regression trees and forests. Electron J Stat 2007;1:519-37.

11. Harrell FEJ. rms: Regression Modeling Strategies. R package version 4.1-3. 2014. Available online: http:// CRAN.R-project.org/package $=$ rms

12. Hosmer DW, Lemeshow S, Sturdivant RX. Applied logistic regression. Third edition. Hoboken: Wiley, 2013:500.

13. Yoshida S, Fukushima S, Miyagawa S, et al. Mitral valve structure in addition to myocardial viability determines the outcome of functional mitral regurgitation after coronary artery bypass grafting. Circ J 2017;81:1620-7.

14. Lancellotti P, Tribouilloy C, Hagendorff A, et al.

Recommendations for the echocardiographic assessment

Cite this article as: Manzan E, Azzolina D, Gregori D, Bizzotto E, Colli A, Gerosa G. Combining echocardiographic and anatomic variables to predict outcomes of mitral valve repair with the NeoChord procedure. Ann Cardiothorac Surg 2021;10(1):122-130. doi: 10.21037/acs-2020-mv-96 of native valvular regurgitation: an executive summary from the European Association of Cardiovascular Imaging. Eur Heart J Cardiovasc Imaging 2013;14:611-44.

15. Colli A, Bizzotto E, Manzan E, et al. Patient-specific ventricular access site selection for the NeoChord mitral valve repair procedure. Ann Thorac Surg 2017;104:e199-202.

16. R Core Team. R: A Language and Environment for Statistical Computing. Vienna: R Foundation for Statistical Computing, 2015.

17. Therneau T. A Package for Survival Analysis in S. version 2.38. 2015. Reference Source 2016.

18. Colli A, Adams D, Fiocco A, et al. Transapical NeoChord mitral valve repair. Ann Cardiothorac Surg 2018;7:812-20.

19. Colli A, Bagozzi L, Banchelli F, et al. Learning curve analysis of transapical NeoChord mitral valve repair. Eur J Cardiothorac Surg 2018;54:273-80.

20. Gerosa G, Nadali M, Longinotti L, et al. Transapical offpump echo-guided mitral valve repair with neochordae implantation mid-term outcomes. Ann Cardiothorac Surg 2021;10:131-40. 